\title{
A Modified Volterra Series Approach for Nonlinear Dynamic Systems Modeling
}

\author{
Domenico Mirri, Member, IEEE, Gaetano Iuculano, Member, IEEE, Fabio Filicori, Member, IEEE, \\ Gaetano Pasini, Member, IEEE, Giorgio Vannini, Member, IEEE, and Gabriella Pellegrini Gualtieri
}

\begin{abstract}
This paper describes a modeling approach for nonlinear dynamic systems based on a modified Volterra series; by comparing the truncation error of this series with that of the classical Volterra one, we outlined that, under the assumption of short-term nonlinear memory effects, the modified series enables a single-fold nonlinear convolution integral to be adopted also in the presence of strong nonlinearities. The measurement-based identification of the first terms of the modified Volterra series is described; experimental and simulation results which confirm the theoretical considerations are also provided.
\end{abstract}

Index Terms-Nonlinear dynamic model, nonlinear system, Volterra series.

\section{INTRODUCTION}

$\mathbf{I}^{\mathrm{N}}$ N MANY application fields, "black box" modeling of nonlinear dynamic systems (i.e., in the absence of a detailed microscopic description of the system structure) is important for the experiment-based characterization and performance evaluation. Such a modeling, however, is quite a complex task owing to the simultaneous presence of both "memory" and nonlinear effects [1]. In fact, when nonlinear effects are negligible (or equivalently the input signal amplitude is small enough) a complete system characterization can be obtained by using the transfer function, which can be easily identified (e.g., by using vector voltmeters under sinusoidal operations) and mathematically modeled (e.g., rational functions with constant coefficients). Conversely, when memory effects are negligible (or, equivalently, the signal bandwidth is small enough), the system transcharacteristic can easily be deduced through a sequence of low-frequency or "static" (i.e., dc) measurements and then described in terms of general-purpose approximating functions (e.g., polynomials or, preferably, "splines" for strong nonlinearities). Unfortunately, when both nonlinear and dynamic "memory" effects must be taken into account simultaneously, a general-purpose "black-box" mathematical description becomes necessarily very complex and almost impossible to use in practice since the set of possible system behaviors is extremely wide since includes any possible system response. Thus, in practice, some approximations and limitations, related to specific properties and/or operating conditions

Manuscript received May 31, 2000; revised January 30, 2001. This paper was recommended by Associate Editor M. Gilli.

D. Mirri, F. Filicori, G. Pasini, and G. P. Gualtieri are with the Dipartimento di Ingegneria Elettrica, University of Bologna, Bologna 40136, Italy.

G. Iuculano is with the Department of Electronic Engineering, University of Florence, Florence 50139, Italy.

G. Vannini is with the Faculty of Engineering, Dipartimento di Ingegneria,

University of Ferrara, Ferrara 44100, Italy

Publisher Item Identifier 10.1109/TCSI.2002.801239. of the system, must be introduced. For instance, the Volterra series [1]-[4], which is in principle capable of describing a very large class of nonlinear systems with memory, can be practically used only for mildly nonlinear systems (or, equivalently, for limited signal amplitude in strongly nonlinear systems), where the kernels of higher order (typically greater than three) can be neglected [5]-[9]. Thus, the truncated Volterra series represents a general-purpose mathematical description for systems under mildly nonlinear conditions, regardless of the system memory and the signal bandwidth. However, in some cases, the most likely operating conditions to be considered for a system are somehow complementary to those which enable the Volterra series truncation, in the sense that nonlinear phenomena are strong, while the nonlinear memory effects are quite limited (unwanted effects). This happens, for instance, in electron devices, where nonlinearity is a "desired" effect, while memory is an "unwanted" side effect.

A modified Volterra-like approach was recently introduced as a viable, interesting alternative to the conventional one [10]-[12]. This approach is based on a series of nonlinear multifold convolution integrals which, unlike the conventional Volterra integrals, are expressed in terms of "dynamic deviations" of the input signal. This series can be truncated to low-dimension terms (e.g., to single- or two-fold integrals) not only for weak but also for relatively strong nonlinearities, provided that the duration of the nonlinear "memory" effects is "short" enough with respect to the signal period.

In this paper, the basic features of this modified Volterra series are outlined (Section II) and the truncation errors are studied in comparison with the classical Volterra formulation (Section III). Measurement-based identification of the first terms of the modified Volterra series is described in Section IV, while simulation results which confirm the theoretical considerations on the truncation error are presented in Section V.

\section{MOdIFIED VOLTERRA SERIES}

The output signal $u(t)$ of a nonlinear dynamic system with a practically finite memory time (strictly speaking a "vanishing" memory) can be described by a certain functional ${ }^{1}$ or a linefunction of its input signal $s(t-\tau)$ in the memory interval $\left(-T_{A} \leq \tau \leq+T_{B}\right)$, where $\tau$ is the shift with respect to the instant $t$ when the output is evaluated

$$
u(t)=F \mid\left[\begin{array}{c}
+T_{B} \\
s-\tau)] \mid \\
-T_{A}
\end{array}\right.
$$

${ }^{1} \mathrm{~A}$ functional [12] is a real-valued function whose domain is a set of real functions. A simple example of functional, on the set of integrable real functions defined on a prefixed domain $D$, is the integral on $D$. 
where $T_{A}$ is identically null in a nonanticipative system. To represent the functional, we use the original symbolism introduced by Volterra [13] and, coherently, we express the functional description of the output signal $u(t)$ through "a series with memory" [3], [4], [12]

$$
u(t)=y_{0}+\sum_{n=1}^{\infty} y_{n}(t)
$$

where $y_{0}$ is a constant independent of the input signal and

$$
y_{n}(t)=\int \begin{gathered}
+T_{B} \\
\cdots \\
\text { with } n \geq 1
\end{gathered}
$$

is the $n$ th-dimensional Volterra term. Each term $y_{n}(t)$ is therefore expressed by means of a multifold convolution integral over the finite memory interval. The weight $h_{n}\left(\tau_{1}, \tau_{2}, \ldots, \tau_{n}\right)$ is the $n$ th-order time-domain Volterra kernel of the system; each kernel is bounded and a symmetrical function of its arguments. The set of kernels describes the dynamic characteristics of the nonlinear system. Equations (2) and (3) can be interpreted as an extension of the well-known linear convolution integral, which describes the time-domain response of a linear dynamic system $(n=1)$, to the nonlinear operation $(n>1)$.

By introducing the dynamic deviation $e(t, \tau)$

$$
e(t, \tau)=s(t-\tau)-s(t)
$$

which represents the deviation of the signal $s(t-\tau)$ with respect to $s(t)$, the output signal at the instant $t$ can be expressed by

$$
u(t)=F \mid\left[\begin{array}{c}
+s(t)+e(t, \tau)] \\
-T_{A}
\end{array}\right.
$$

Thus, the following dynamic-deviation-based Volterra series [9]-[11] can be introduced:

$$
u(t)=z_{0}(t)+\sum_{n=1}^{+\infty} z_{n}(t)
$$

where $z_{0}(t)$ is the system response when the dynamic deviations are identically null, i.e., it represents the response of the nonlinear dynamic system to a dc input equal to $s(t)$ and is a purely algebraic function of $s(t)$

$$
z_{0}(t)=f[s(t)]=F \underset{-T_{B}}{\left[\begin{array}{c}
T_{B} \\
-T_{A}
\end{array}\right]}
$$

The memory effects in (6) are accounted for by the sum of terms

$$
z_{n}(t)=\int \begin{gathered}
+T_{B} \\
\cdots \\
\text { with } n \geq 1
\end{gathered}
$$

which represent the $n$ th-dimensional modified Volterra terms expressed as a multiple integral of the product of $r$ dynamic de- viations in the memory interval, evaluated with respect to $n$ different shifts $\tau_{1}, \ldots \tau_{n}$ and weighted by the $r$ th-order modified Volterra kernel $g_{r}\{\cdot\}$. Each kernel is generally a nonlinear function of the reference signal $s(t)$. It can be shown (see the Appendix) that each term of the modified series can be expressed as a function of the original Volterra kernels through the relationships (39) and (43). From these equations we deduce that the original Volterra series (2) is a particular case of the modified Volterra one (6) since it can be obtained from (6) by imposing $s(t)=0$.

From the previous considerations, it is clear that both the conventional Volterra series and the modified one have the same asymptotic convergence properties. However, when for practical reasons only a relatively small, finite number of terms must be considered, the basic properties of the two series are quite different. For instance, when both the series are truncated to the single-fold integral, the conventional Volterra model corresponds to a linear convolution (i.e., a purely linear dynamic system), while the modified one is capable of describing not only nonlinear systems without memory through the term $z_{0}(t)$, but also some nonlinear dynamic effects represented by the single-fold convolution integral. This happens since the kernels in the modified series are nonlinearly dependent on the instantaneous value of the input $s(t)$. Thus, an adequate comparison of the two series should be based on the study of the accuracy properties in the presence of quite a limited, practically usable, number of terms.

A qualitative comparison between the Volterra series and the modified one can be made by referring to the periodic signal $s(t-\tau)$ reported in Fig. 1(a) as a function both of the time $t$ and the shift $\tau$. The contributions of the successive products, which appear in (3), of the shifted signals of Fig. 1(a) may turn out to be quite large also in the presence of a short memory interval. Instead, the dynamic deviations $e(t, \tau)$ can be small even in the presence of large values of the input signal, provided that the memory interval is sufficiently short [Fig. 1(b)]. Under these conditions, the contributions of the successive products in (8) become progressively less important. In other words, we can characterize the system with a small number of terms using the Volterra series only in the presence of a small amplitude signal, independently of the memory interval; instead, we can represent the output signal with a small number of terms using the modified Volterra series also in the presence of large amplitude signals if the memory interval is sufficiently short. In the next section, these considerations are mathematically outlined.

\section{TRuncation ERrors of the VolterRa SERIES AND THE MODIFIED ONE}

In the following, the convergence properties of the conventional Volterra series and the modified one will be studied and compared. In particular, as far as the conventional series is concerned (2) and (3), we can write

$$
\begin{aligned}
u(t)=y_{0}+\sum_{n=1}^{m} \int \begin{array}{c}
+T_{B} \\
-T_{A}
\end{array} & h_{n}\left(\tau_{1}, \tau_{2}, \ldots, \tau_{n}\right) \\
& \cdot \prod_{i=1}^{n}\left[s\left(t-\tau_{i}\right) d \tau_{i}\right]+\Delta_{m}
\end{aligned}
$$




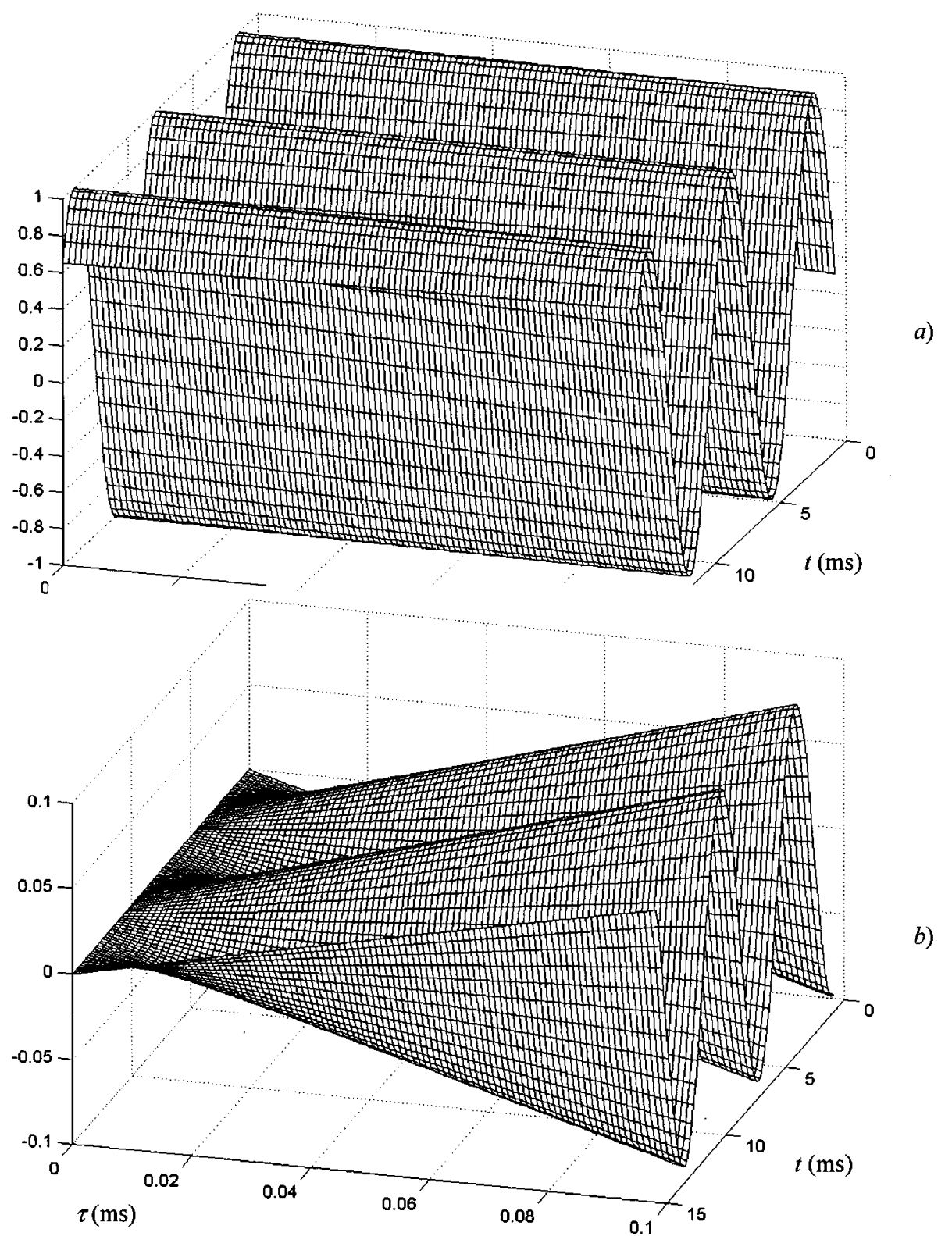

Fig. 1. (a) Input signal $s(t-\tau)$. (b) Associated dynamic deviation $e(t, \tau)$ plotted as a function both of the time $t$ and the shift $\tau$.

where $\Delta_{m}$ is the remainder of the series truncated to the $m$ th-order term

$$
\begin{array}{r}
\Delta_{m}=\sum_{n=m+1}^{\infty} \int \begin{array}{c}
\cdots \\
-T_{A}
\end{array} \quad \cdot \int h_{n}\left(\tau_{1}, \tau_{2}, \ldots, \tau_{n}\right) \\
\cdot \prod_{i=1}^{n}\left[s\left(t-\tau_{i}\right) d \tau_{i}\right] .
\end{array}
$$

Moreover, taking into account well-known properties of the absolute values of sums and integrals, we have

$$
\begin{array}{r}
\left|\Delta_{m}\right| \leq \sum_{n=m+1}^{\infty} \int \begin{array}{c}
\ldots \\
-T_{A}
\end{array} \quad \cdots \int\left|h_{n}\left(\tau_{1}, \tau_{2}, \ldots, \tau_{n}\right)\right| \\
\cdot \prod_{i=1}^{n}\left[\left|s\left(t-\tau_{i}\right)\right| d \tau_{i}\right]
\end{array}
$$

and, by indicating with $S_{\max -}$ the maximum absolute amplitude of the input signal in the memory interval, i.e., $S_{\max _{\tau}}=$ $\max _{\tau}|s(t-\tau)|$ for $-T_{A} \leq \tau \leq+T_{B}$, we can write

$$
\left|\Delta_{m}\right| \leq \sum_{n=m+1}^{\infty} S_{\max _{\tau}}^{n} \int{ }_{-T_{A}}^{+T_{B}} \quad \ldots \int\left|h_{n}\left(\tau_{1}, \tau_{2}, \ldots, \tau_{n}\right)\right| \prod_{i=1}^{n} d \tau_{i} .
$$

We introduce now the positive parameter $B_{n}$ defined as follows:

$$
\begin{aligned}
& \left(B_{n}\right)^{n-1}=\int \begin{array}{cc}
\cdots & \cdots \\
-T_{A} & \cdots
\end{array}\left|h_{n}\left(\tau_{1}, \tau_{2}, \ldots, \tau_{n}\right)\right| \prod_{i=1}^{n} d \tau_{i}, \\
& \text { for } n>1 \text {. }
\end{aligned}
$$

This parameter is evaluated through the multiple integral of the absolute Volterra kernel of the same dimension $n$ and represents 
an equivalent amplitude of the $n$ th-order nonlinear effects. If these equivalent amplitudes have an upper limit, we can introduce their maximum

$$
B_{\max }=\max _{n}\left[B_{n}\right] .
$$

By substituting (13) and (14) in (12), taking into account the properties of the geometric series under the hypothesis that $S_{\max \tau} B_{\max }<1$, we can write

$$
\begin{aligned}
\left|\Delta_{m}\right| & \leq \sum_{n=m+1}^{\infty} S_{\max \tau}^{n} B_{\max }^{n-1} \\
& =\frac{1}{B_{\max }} \sum_{n=m+1}^{\infty}\left(S_{\max _{\tau}} B_{\max }\right)^{n} \\
& =\frac{1}{B_{\max }}\left[\sum_{n=0}^{\infty}\left(S_{\max _{\tau}} B_{\max }\right)^{n}-\sum_{n=0}^{m}\left(S_{\max _{\tau}} B_{\max }\right)^{n}\right] \\
& =\frac{S_{\max _{\tau}}^{m+1} B_{\max }^{m}}{1-S_{\max _{\tau}} B_{\max }} .
\end{aligned}
$$

Therefore, the superior limit of the absolute truncation error of the Volterra series, which becomes negligible when $\left(S_{\max _{\tau}} B_{\max }\right)^{m} \ll 1$, depends on the joint contribution of the maximum equivalent amplitude $B_{\max }$ of the nonlinear effects of the system and the maximum amplitude $S_{\max _{\tau}}$ of the input signal. More precisely, (15) shows that, in order to keep the truncation error within sufficiently small values, for a given system characterized by a given value of $B_{\max }$, it may be necessary to limit the maximum amplitude $S_{\max _{\tau}}$ of the input signal in the memory interval. In practical cases, the Volterra series must be truncated to the third- or fourth-order terms since it is very difficult to identify higher order terms [5]-[8]. Thus, (15) implies that, for accuracy reasons, the signal amplitude must be small enough to guarantee mildly nonlinear operation even when the input signal bandwidth is so small that the "memory effects" in the system are of a relatively short duration, or even negligible.

The truncation error properties of the modified series, instead, are quite different since the integral series expansion is expressed in terms of the dynamic deviations $e(t, \tau)=s(t-$ $\tau)-s(t)$ instead of simply $s(t)$. This leads to more complex kernels [as they are nonlinearly dependent on the present signal value $s(t)$ ]; however, this greatly increases the "descriptive capabilities" of the first terms of the modified series. In fact, the term $z_{0}(t)$ in (6) clearly describes a nonlinear system without memory, while in the conventional Volterra series the corresponding term is not normally considered as it would simply be a constant [4]. Analogously, while the single-fold integral in the conventional Volterra series (3) simply describes a purely linear dynamic system, the corresponding one in (8) describes a nonlinear system with memory through a linear convolution nonlinearly controlled by $s(t)$. Thus, the modified Volterra series truncated to the single-fold integral term can be used for the modeling of some types of nonlinear dynamic systems. More precisely, as will be shown in the following and in accord to the examples provided in Section V, the modified series can be truncated to $n=1$ when dealing with nonlinear systems whose operating conditions satisfy a "short term" memory constraint In such cases, kernel identification becomes quite easy, since it can be carried out directly on the basis of conventional dc measurements and small-signal bias-dependent ac parameters (Section IV).

In order to evaluate the truncation error of the modified Volterra series, (6) can be conveniently rewritten, by taking into account (8), in this form

$$
u(t)=z_{0}(t)+\int_{-T_{A}}^{+T_{B}} g_{1}\left\{s(t), \tau_{1}\right\} e\left(t, \tau_{1}\right) d \tau_{1}+\Delta
$$

where $\Delta$ is the remainder of the series truncated to the singledimensional term, that is

$$
\Delta=\sum_{n=2}^{+\infty} \int \underset{-T_{A}}{\cdots} \cdots \int g_{n}\left\{s(t), \tau_{1} \cdots \tau_{n}\right\} \prod_{i=1}^{n} e\left(t, \tau_{i}\right) d \tau_{i} .
$$

We can quite reasonably assume that $s(t-\tau)$ is differentiable with respect to $\tau$ in the memory interval; this is true for finite bandwidth signals (for example this excludes strictly "ideal" square or triangular waveforms but includes the corresponding "real" ones). By expressing the dynamic deviations in the Lagrange form, we can rewrite (4) as

$$
e\left(t, \tau_{i}\right)=s\left(t-\tau_{i}\right)-s(t)=-\tau_{i}\left[\frac{d s(t-\tau)}{d \tau}\right]_{\tau=\vartheta \tau_{i}}
$$

with $0<\vartheta<1$. By substituting into (17) we obtain

$$
\begin{aligned}
\Delta=\sum_{n=2}^{+\infty}(-1)^{n} \int \begin{array}{c}
\cdots \\
-T_{A}
\end{array} & \cdots \int g_{n}\left\{s(t), \tau_{1} \cdots \tau_{n}\right\} \\
& \cdot \prod_{i=1}^{n} \tau_{i}\left[\frac{d s(t-\tau)}{d \tau}\right]_{\tau=\vartheta \tau_{i}} d \tau_{i} .
\end{aligned}
$$

For well-known properties of the absolute values of sums and integrals, we have

$$
\begin{aligned}
& |\Delta| \leq \sum_{n=2}^{+\infty} \mid \int \underset{-T_{A}}{{ }^{+T_{B}}} \quad \cdots \int g_{n}\left\{s(t), \tau_{1} \cdots \tau_{n}\right\} \prod_{i=1}^{n} \tau_{i} \\
& \cdot\left[\frac{d s(t-\tau)}{d \tau}\right]_{\tau=\vartheta \tau_{i}} d \tau_{i}
\end{aligned}
$$

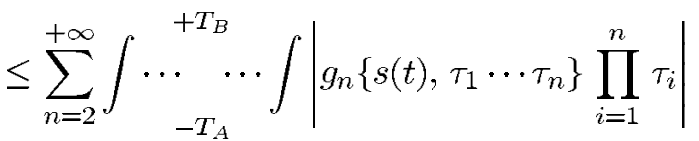

$$
\begin{aligned}
& \cdot\left|\prod_{i=1}^{n}\left[\frac{d s(t-\tau)}{d \tau}\right]_{\tau=\vartheta \tau_{i}}\right| \prod_{i=1}^{n} d \tau_{i} .
\end{aligned}
$$

By introducing the maximum of the signal derivative within the memory time interval $\left(+T_{B},-T_{A}\right)$

$$
\left|\frac{d s(t-\tau)}{d \tau}\right|_{\max _{\tau}}=\max _{\tau}\left[\left|\frac{d s(t-\tau)}{d \tau}\right|_{\tau=\vartheta \tau_{i}}\right]
$$


(20) can be rewritten as follows:

$$
\begin{aligned}
|\Delta| \leq & \left.\sum_{n=2}^{+\infty} \int \begin{array}{c}
+T_{B} \\
-T_{A}
\end{array}\right]\left|g_{n}\left\{s(t), \tau_{1} \cdots \tau_{n}\right\} \prod_{i=1}^{n} \tau_{i}\right| \\
\cdot\left(\left|\frac{d s(t-\tau)}{d \tau}\right|_{\max _{\tau}}\right) & \prod_{i=1}^{n} d \tau_{i} \\
= & \sum_{n=2}^{+\infty}\left(\left|\frac{d s(t-\tau)}{d \tau}\right|_{\max _{\tau}}\right)^{n} \int \cdots \quad \cdots \int \\
\cdot & g_{n}\left\{s(t), \tau_{1} \cdots \tau_{n}\right\}\left|\prod_{i=1}^{n}\right| \tau_{i} \mid \prod_{i=1}^{n} d \tau_{i} .
\end{aligned}
$$

As far as the nonlinear dynamic characteristics of the system are concerned, we can define the parameters $T_{n}\{\cdot\}$ and $C_{n}\{\cdot\}$. The first one, which has the physical dimension of time, is defined as follows:

$$
\begin{aligned}
& \left(T_{n}\{s(t)\}\right)^{n} \\
& =\frac{\int{ }_{-T_{A}}^{+T_{B}} \boldsymbol{\cdots}\left|\sin \left\{s(t), \tau_{1}, \ldots \tau_{n}\right\} \prod_{i=1}^{n} \tau_{i}\right| \prod_{i=1}^{n} d \tau_{i}}{\int \begin{array}{c}
+T_{B} \\
-T_{A}
\end{array}}
\end{aligned}
$$

and can be interpreted as an equivalent time-duration of the $n$ th-dimensional kernel. The second parameter is positive and defined as follows:

$$
\begin{aligned}
& \left(C_{n}\{s(t)\}\right)^{n-1}=\int \underset{-T_{A}}{\ldots} \quad \ldots \int\left|g_{n}\left\{s(t), \tau_{1}, \ldots \tau_{n}\right\}\right| \prod_{i=1}^{n} d \tau_{i}, \\
& \text { with } n>1 \text {. }
\end{aligned}
$$

It takes into account the equivalent amplitude of the purely dynamic nonlinear effects of order $n$ through the multiple integral of the absolute kernel of the same dimension. By recalling (13) and (43), we can deduce that, for $s=0$, the term $C_{n}\{0\}$ of the modified series coincides with the corresponding term $B_{n}$ of the conventional one.

By considering (23) and (24), the upper bound on the truncation error defined by (22) can be rewritten as follows:

$$
|\Delta| \leq \sum_{n=2}^{+\infty}\left(\left|\frac{d s(t-\tau)}{d \tau}\right|_{\max _{\tau}} T_{n}\{s(t)\}\right)^{n}\left(C_{n}\{s(t)\}\right)^{n-1} .
$$

Under the hypothesis that both the parameters $T_{n}\{\cdot\}$ and $C_{n}\{\cdot\}$ have an upper limit

$$
T_{\max }=\max _{\substack{n \geq 2 \\ s(t)}}\left[T_{n}\{s(t)\}\right] \quad C_{\max }=\max _{\substack{n \geq 2 \\ s(t)}}\left[C_{n}\{s(t)\}\right]
$$

where $T_{\max }$ is the maximum equivalent memory time-duration of the purely dynamic nonlinear effects associated to the re- mainder of the series and $C_{\max }$ the corresponding maximum equivalent amplitude, (25) becomes

$$
|\Delta| \leq \sum_{n=2}^{+\infty} \frac{\left(\left|\frac{d s(t-\tau)}{d \tau}\right|_{\max _{\tau}} T_{\max } C_{\max }\right)^{n}}{C_{\max }} .
$$

In order to emphasize the relationship between signal amplitude, frequency, ${ }^{2}$ shape, and the corresponding truncation error, the positive adimensional peak-derivative shape-factor $\rho_{f}$ is introduced

$$
\rho_{f}=\frac{\left|\frac{d s(t-\tau)}{d \tau}\right|_{\max _{\tau}}}{f_{1} S_{P P}}
$$

where $S_{P P}$ and $f_{1}$ represent the peak-to-peak amplitude of the signal and its fundamental frequency, respectively. By substituting into (27), we obtain

$$
|\Delta| \leq \sum_{n=2}^{+\infty} \frac{\left(\rho_{f} f_{1} S_{P P} T_{\max } C_{\max }\right)^{n}}{C_{\max }} .
$$

By indicating with $b$ the following quantity:

$$
b=\rho_{f} f_{1} T_{\max } S_{P P} C_{\max }
$$

taking into account the properties of the geometric progressions and assuming ${ }^{3} 0<b<1$, we obtain the final relationship

$$
\begin{aligned}
|\Delta| & \leq \frac{1}{C_{\max }}\left[\sum_{n=0}^{+\infty} b^{n}-b-1\right] \\
& =\frac{1}{C_{\max }}\left[\frac{1}{1-b}-(1+b)\right] \\
& =\frac{\left(\rho_{f} f_{1} T_{\max } S_{P P}\right)^{2} C_{\max }}{1-\rho_{f} f_{1} T_{\max } S_{P P} C_{\max }} .
\end{aligned}
$$

Therefore, the truncation properties of the dynamic-deviationbased series depend basically on the product of the maximum equivalent amplitude $C_{\max }$ of the nonlinear purely dynamic effects and the quantity $\rho_{f} f_{1} T_{\max } S_{P P}$. It is important to point out that in this case the upper limit of the truncation error depends, for a given signal shape (i.e., for a given $\rho_{f}$ ), not only on the maximum peak-to-peak signal amplitude $S_{P P}$ and on the maximum equivalent amplitude $C_{\max }$ of the purely dynamic nonlinear effects, but also on the product between the correspondent maximum equivalent time-duration $T_{\max }$ of the nonlinear effects in the system and the input signal frequency $f_{1}$. Therefore, this series can be truncated to the one-dimensional kernel even in the presence of strong nonlinear effects provided that the product $f_{1} T_{\max }$ is small enough for a given shape-factor $\rho_{f}$. In particular, it should be emphasized that, in the dynamic-deviation-based Volterra series, the truncation error depends, for a given system, not only on the amplitude of the applied signal, as in the conventional Volterra description, but on a trade off between its peak-to-peak value and fundamental frequency for a

${ }^{2}$ For simplicity, a periodic signal $s(t)$ with fundamental frequency $f_{1}$ is considered in the following.

${ }^{3}$ The condition $0<b<1$ is necessary for the convergence of the modified Volterra series. 


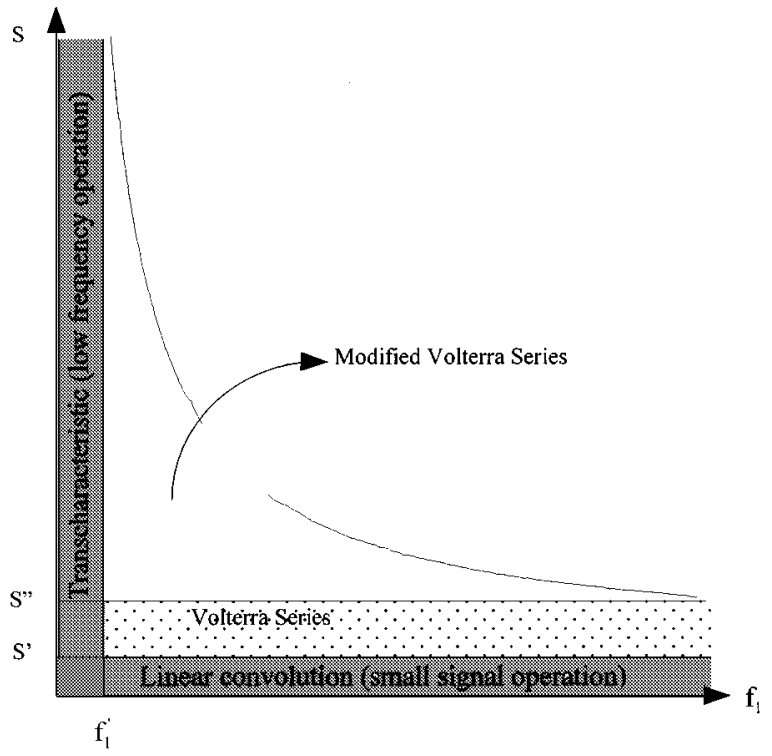

Fig. 2. Qualitative description of the region of validity of the Volterra series and the modified one truncated to the first terms.

given "shape" (or equivalently its bandwidth). The upper limit of the truncation error in the Volterra series is instead dependent only on the signal amplitude and, unfortunately, is not necessarily small in the presence of low-bandwidth signals.

The different behavior of the two series when only a limited number of terms is used can be qualitatively described by Fig. 2, where the regions of validity 4 of both the truncated Volterra series and the truncated modified one are schematically represented in the space of the signal amplitude $S$ and its fundamental frequency $f_{1}$. In particular, the area below the line $S=S^{\prime \prime}$ symbolically represents the validity region of the truncated conventional Volterra series since, according to (15), in order to guarantee, for a given system (i.e., given $B_{\max }$ ), a given upper limit on the truncation error $\Delta_{m}$, an upper limit on the signal amplitude $S_{\max } \leq S^{\prime \prime}$ must be imposed. In particular, for $m=1$ the conventional Volterra series simply becomes a single-fold linear convolution, which can only be used under the so called "small signal" operating conditions (see the area below the line $S=$ $S^{\prime}$ ). Instead, the validity region for the modified Volterra series with $m=1$ is symbolically represented by the "hyperbolic" region below the solid line in Fig. 2. In fact, for a given system (i.e., given $T_{\max }$ and $C_{\max }$ ), for a constant shape factor $\rho_{f}$ of the signal and by reasonably assuming $b<1$, (31) can be approximated by $|\Delta| \leq k\left(f_{1} S_{P P}\right)^{2}$ (where $k$ is a constant), which shows that the constant truncation error locii have an upper limit defined by a hyperbolic function in the $S_{P P} / f_{1}$ space. In particular, for $m=0$ the modified Volterra series becomes a purely "static" nonlinear transcharacteristic $z_{0}(t)$, whose validity is clearly limited to low-frequency operations (area limited by the line $f_{1}=f_{1}^{\prime}$ in Fig. 2). Clearly, the conventional Volterra series is preferable for mildly nonlinear systems with strong memory effects (i.e., relatively high operating frequencies). The modified series, instead, is usable also in strongly nonlinear operation provided that memory effects in the system

${ }^{4}$ That is, the regions where the truncation error is within a given limit. are relatively short with respect to the signal frequency (i.e. small $T_{\max } f_{1}$ product). This condition is satisfied, for instance, in electron devices described in a voltage-controlled form (possibly after parasitic de-embedding 5 ), or in S/H-ADC devices (eventually after suitable modifications in the system description) [14]. In such cases, the modified Volterra series can be truncated [see (16)] to the first convolution integral. Moreover, (16) shows an interesting analogy with the small-signal description of a system. In fact, the short-term memory concept (i.e., small dynamic deviations) enables the description in terms of a single convolution integral with respect to the dynamic deviations to be adopted even under large signals. Likewise, the small-signal hypothesis allows for the description in terms of a convolution integral with respect to the small signals applied.

\section{MEASUREMENT-BASED IDENTIFICATION OF THE TRUNCATED MOdified VOLTERRA SERIES}

The identification of the modified Volterra series truncated to the first terms (16)

$$
\begin{aligned}
u(t) & \cong z_{0}(t)+z_{1}(t) \\
& =z_{0}(t)+\int_{-T_{A}}^{+T_{B}} g_{1}\left\{s(t), \tau_{1}\right\}\left[s\left(t-\tau_{1}\right)-s(t)\right] d \tau_{1}
\end{aligned}
$$

can easily be carried-out by means of frequency-domain measurements. To this end, we consider a discrete spectrum signal $s(t)$, described by the generalized Fourier series

$$
s(t)=\sum_{q=-N}^{+N} S_{q} e^{j 2 \pi f_{q} t}
$$

where $S_{-q}=S_{q}^{*}, f_{-q}=-f_{q}$ and $f_{0}=0$; by introducing the Fourier transform $G_{1}\{s(t), f\}$ of the first-order kernel $g_{1}\left\{s(t), \tau_{1}\right\}$ for a system with limited memory duration $T_{a}$

$$
G_{1}\{s(t), f\}=\int_{-T_{A}}^{+T_{B}} g_{1}\left\{s(t), \tau_{1}\right\} e^{-j 2 \pi f \tau_{1}} d \tau_{1}
$$

the term $z_{1}(t)$ can be expressed as follows:

$$
\begin{gathered}
z_{1}(t)=\sum_{q=-N}^{+N} S_{q} e^{j 2 \pi f_{q} t}\left[\int_{-T_{A}}^{+T_{B}} g_{1}\left\{s(t), \tau_{1}\right\} e^{-j 2 \pi f_{q} \tau_{1}} d \tau_{1}\right. \\
\left.-\int_{-T_{A}}^{+T_{B}} g_{1}\left\{s(t), \tau_{1}\right\} d \tau_{1}\right] \\
=\sum_{\substack{q=-N \\
q \neq 0}}^{+N} D\left\{s(t), f_{q}\right\} S_{q} e^{j 2 \pi f_{q} t}
\end{gathered}
$$

where

$$
D\{s(t), f\}=G_{1}\{s(t), f\}-G_{1}\{s(t), 0\}
$$

5"Intrinsic" electron devices (i.e., devices not affected by relevant parasitic effects) are characterized by very short memory effects. This is justified both by experimental and simulated results [10], [11], [16] and is coherent with the description of device behavior in terms of quasistatic voltage-controlled charges. 
with $D\{s(t),-f\}=D^{*}\{s(t), f\}$ and $D\{s(t), 0\}=0$. Therefore, the truncated modified Volterra series of (32) can be expressed [14] in the form (7)

$$
u(t) \cong f[s(t)]+\sum_{q=-N}^{+N} D\left\{s(t), f_{q}\right\} S_{q} e^{j 2 \pi f_{q} t} .
$$

The quantity $D\{\cdot\}$ represents a nonlinear purely dynamic function which weights the contribution to the output of each spectral component of the input signal and depends both on the instantaneous value and frequency of the input signal. Equation (37) is another, more convenient, way of representing the proposed nonlinear dynamic model defined by (6)-(8), when the integral series is truncated to the first, single-fold integral. Moreover, (37) provides a straightforward way of computing the system output to any input signal $s(t)$, defined by a given set of discrete spectral components $S_{q}$, once the algebraic function $f[s(t)]$ and the nonlinearly controlled transfer function $G_{1}\{s(t), f\}$ have been measured for any frequency $f$ and any input signal value $s$ over the region of possible operating conditions of the system to be modeled. To this end, a suitable identification experimental procedure, based on bias-dependent small-signal measurements, can be defined by using (37). In fact, by considering an input test signal made up of a dc bias component $S_{0}$ and a superimposed small sinusoidal signal with amplitude $S_{1}$ and frequency $f_{r}=-f_{-r}$, by expanding (37) in the Taylor series with respect to the variable $s=s(t)$ around $S_{0}$ and by assuming the system to be linear with respect to the sinusoidal component, we can write [14]

$$
\begin{aligned}
u(t) \cong & f\left(S_{0}\right)+\sum_{r=-1,+1}\left[\left(\frac{d z_{0}(s)}{d s}\right)_{s=S_{0}}+D\left\{S_{0}, f_{r}\right\}\right] \\
& \cdot S_{r} e^{j 2 \pi f_{r} t} \\
= & f\left(S_{0}\right)+\sum_{r=-1,+1} A\left\{S_{0}, f_{r}\right\} S_{r} e^{j 2 \pi f_{r} t} .
\end{aligned}
$$

Since, according to (36), $D\left\{S_{0}, 0\right\}=0$, (38) shows that the nonlinear algebraic function $f\left(S_{0}\right)$ can be directly measured by pure dc measurements [i.e., $s(t)=S_{0}$ ] of the static transcharacteristic of the system. Instead, the frequency and signal dependent complex function $D$, which characterizes the nonlinear dynamics of the system, can be completely characterized by measuring, with a vector voltmeter, the bias-dependent small-signal transfer function $A\left\{S_{0}, f_{r}\right\}$. In fact, according to (38), the complex function $D\{s, f\}$ can be directly computed, for any value $s=S_{0}$ and $f=f_{r}$, as

$$
\begin{aligned}
D\left\{S_{0}, f_{r}\right\} & =A\left\{S_{0}, f_{r}\right\}-\left(\frac{d z_{0}(s)}{d s}\right)_{s=S_{0}} \\
& =A\left\{S_{0}, f_{r}\right\}-A\left\{S_{0}, 0\right\}
\end{aligned}
$$

where $A\left\{S_{0}, 0\right\}=\left(d z_{0}(s) / d s\right)_{s=S_{0}}$ clearly represents the low-frequency small-signal transfer function of the system at the bias point $S_{0}$. Thus, on the basis of conventional measurements of the bias-dependent small-signal transfer function $A\left\{S_{0}, f\right\}$, the nonlinearly controlled purely dynamic transfer function $D\left\{S_{0}, f\right\}$ can be easily deduced from a sufficiently large discrete set of bias and frequency values in order to obtain, through a suitable interpolation procedure, the nonlinear purely dynamic transfer function $D\{s(t), f\}$.

The nonlinear dynamic model defined by (37) can be easily implemented in conventional simulation tools. In fact, (37), when the system output $u(t)$ is directly expressed in terms of both time-domain values and spectral components $S_{q}$ of the input signal can be directly embedded within harmonic-balance circuit analysis algorithms (this was done in order to obtain the simulated results described in Section V). However, this is not always possible, since the source code of commercial circuit or systems simulators is normally not accessible to the user. In such cases, nonlinear model (37) or (32) must be somehow approximated with negligible loss of accuracy, in order to allow for model implementation through the typical user tools available in the input interfaces of commercial simulation packages.

For instance, the convolution integral in (32) can be discretized, so that the nonlinear model can be described in terms of elementary delay operators and nonlinear memoryless elements. This approach was adopted for implementing the nonlinear dynamic model of a S/H-ADC device [17].

\section{Simulated Results}

According to the considerations made in the previous section, the identification of the nonlinear model defined by (37) is relatively easy as it can be carried out on the basis of conventional dc and bias-dependent small-signal ac measurements. The model can be used to predict the large-signal dynamic system response provided that the "short memory" condition, which guarantees a sufficiently small truncation error, is verified. Relatively short duration of nonlinear memory phenomena is commonly encountered in devices whose response must be necessarily "fast," as happens for instance in sample-hold devices or electron devices when described in a voltage controlled form. ${ }^{6}$ Thus, the accuracy of the nonlinear dynamic model in (37) [11, nonlinear integral model, (NIM )]) obtained by truncating the modified Volterra series to the first term has been experimentally verified for field-effect transistors operating at microwave frequencies under strongly nonlinear operation [10], [16]-[18]. The extension of the model in (37) to the case of two-port electron devices is straightforward since it is sufficient to consider (37) as a matrix description where $u(t)$ and $s(t)$ are the vectors of port currents and voltages, and $z_{0}\{\cdot\}, H\{\cdot\}$ suitable $2 \times 2$ matrices.

The NIM was identified through the procedure described in Section IV, i.e., by means of conventional measurements (dc characteristic and frequency-bias dependent small-signal ac parameters), and implemented in a harmonic-balance (HB) program for circuit analysis. As an example, Fig. 3 shows the excellent agreement between the measured output power spectrum and the performance predicted by the NIM for an Alcatel-Telettra $0.6 \times 600 \mu \mathrm{m}$ GaAs MESFET $50 \Omega$ amplifier with sinusoidal source at $5 \mathrm{GHz}$.

\footnotetext{
"In fact, in "quasistatic charge-controlled" models, charges stored within the intrinsic device are normally considered as instantaneous, "memoryless" functions of the voltage applied.
} 


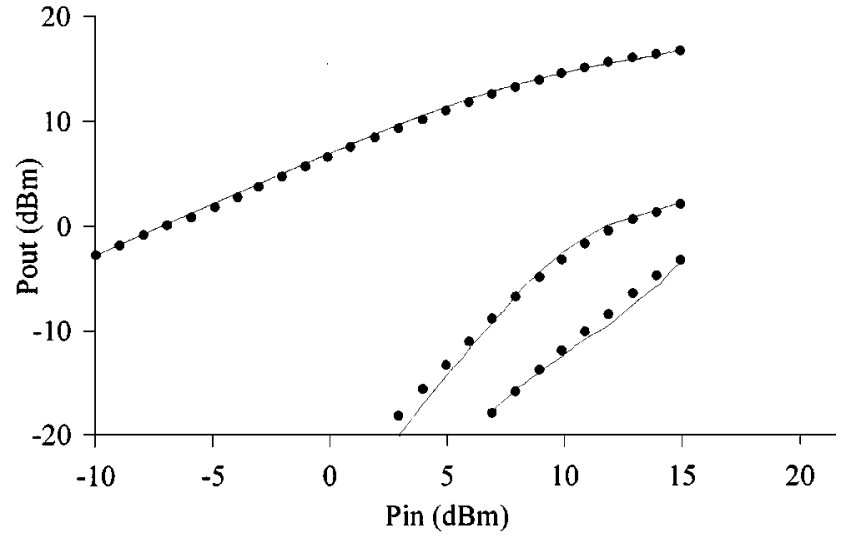

Fig. 3. Comparison between measured $(\cdot)$ and predicted $(-)$ output power spectrum for a $600 \times 0.6 \mu \mathrm{m}$ GaAs MESFET versus input power at $f_{0}=5 \mathrm{GHz}$

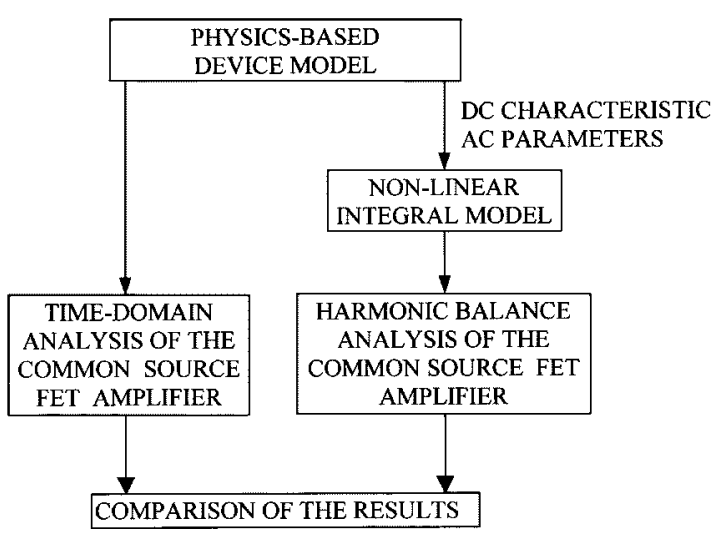

Fig. 4. Flow-chart of the simulation procedures adopted to practically validate the properties of the dynamic-deviation-based Volterra series.

In order to provide a "practical" confirmation of the relevant properties of the dynamic-deviation-based series in terms of the upper limit of the truncation error (31), the following simulated experiment, described by the flow-chart in Fig. 4, has been carried out. A highly accurate two-dimensional (2-D) drift-diffusion simulator was adopted to identify the NIM for a $0.5-\mu \mathrm{m}$ field-effect transistor. As described in [10], [15], and [16], dc characteristics and frequency-bias dependent small-signal ac parameters were accurately computed on a suitable grid of bias conditions and in the chosen frequency range. The identified model was used, in the framework of a microwave circuit analysis program based on HB techniques, to predict the nonlinear dynamic performance of a common-source, 50- $\Omega$ loaded, microwave amplifier driven under strongly nonlinear operation by a large-signal sinusoidal source. The results were compared with the performance predicted for the same amplifier by means of the 2-D drift-diffusion simulator.7 In particular, Fig. 5 shows the constant error loci, associated to maximum discrepancies of $2 \%$ and $5 \%$ between the instantaneous values of the drain

\footnotetext{
${ }^{7}$ The strong difference between the two kinds of simulators should be outlined: the first one is based on the highly accurate numerical solution of the physics-based Poisson's and continuity semiconductor equations, and the second one based on the approximated mathematical black-box model described by (37).
}

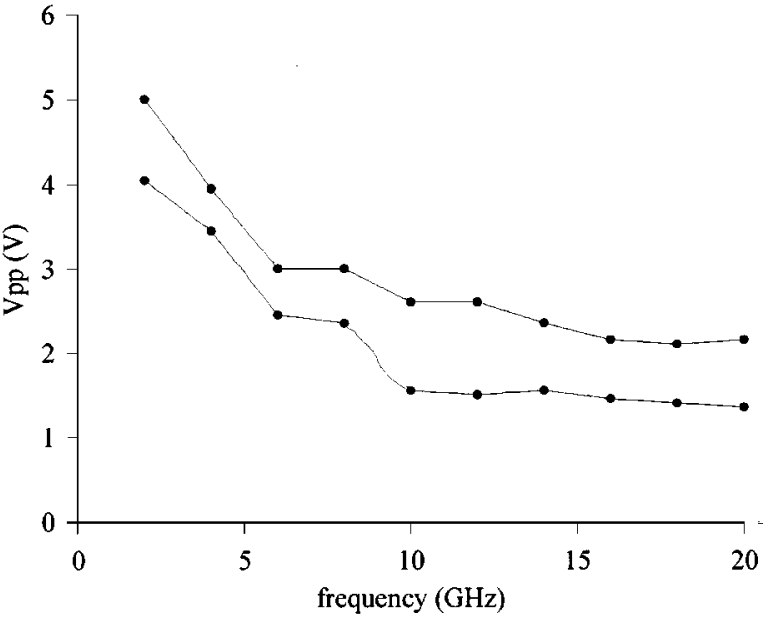

Fig. 5. Constant error loci corresponding to maximum discrepancies between the instantaneous values of the drain currents predicted by the NIM and 2-D drift-diffusion simulations, for a common-source, FET large-signal amplifier.

currents computed by means of the 2-D and HB simulations, plotted as functions of the peak-to-peak gate-to-source voltage $V_{P P}$ and the source frequency. It must be said that the simulations in Fig. 5 correspond to relatively strong nonlinear operation since the associated gain compression (which is conventionally adopted as a measure of the level of nonlinearity) goes from a minimum of $2 \mathrm{~dB}$ at $20 \mathrm{GHz}$ to a maximum of $7 \mathrm{~dB}$ at $2 \mathrm{GHz}$. The errors loci in Fig. 5 are clearly in accordance with the upper absolute limit defined by (31) and shown in Fig. 2. In particular, Fig. 5 points out that the accuracy of the NIM, as predicted by (37), is mainly dependent on a trade off between the peak-to-peak voltage (i.e., $V_{P P}$ ) and the operating frequency (i.e., $f_{1}$ ). In other words, the same level of accuracy can be obtained at higher frequencies if the $V_{P P}$ value of the input voltage (i.e., the level of nonlinearity) is reduced. It can be observed that the constant truncation-error loci associated, for a given system, to the Volterra series would be represented by horizontal lines in the $V_{P P} / f_{1}$ space since the convergence properties of the Volterra series depend only on the input signal amplitude (see Fig. 2).

\section{CONCLUSIONS}

A mathematical approach for the measurement-based modeling of nonlinear dynamic systems having relatively short nonlinear memory effects has been described. It has been derived by truncation of a suitably modified Volterra series expansion obtained by introducing the dynamic deviations of the input signal with respect to its past values. In particular, it has been shown that, unlike the Volterra series, the truncation error can be negligible, even for strongly nonlinear operation, provided that the "short" term nonlinear memory condition is satisfied or, more precisely, an upper limit is imposed on the amplitude-frequency product which characterizes a given input signal. Under this hypothesis model-identification can be easily carried out in the frequency domain on the basis of small signal bias-dependent conventional measurements. The validity of this approach has been preliminary tested in the nonlinear modeling of field-effect transistors both through direct measurements and comparison with 
the results of 2-D numerical device simulations based on accurate physics models. Good agreement was found in all cases with the performance predicted by the mathematical model proposed.

\section{APPENDIX}

By introducing in (3) the dynamic deviation $e(t, \tau)(4)$ and developing the products, the $n$ th-order functional of the Volterra series can be written in the following form [11]:

$$
\begin{aligned}
& y_{n}(t)=\int \ldots h_{n}\left(\tau_{1}, \tau_{2}, \ldots, \tau_{n}\right) \\
& \cdot \prod_{i=1}^{n}\left[e\left(t, \tau_{i}\right)+s(t)\right] \prod_{i=1}^{n} d \tau_{i} \\
& =s^{n}(t) \int \ldots h_{-T_{A}} h_{n}\left(\tau_{1}, \tau_{2}, \ldots, \tau_{n}\right) \prod_{i=1}^{n} d \tau_{i} \\
& +\sum_{r=1}^{n} s^{n-r}(t)\left(\begin{array}{l}
n \\
r
\end{array}\right) \int \cdots \underbrace{+T_{B}}_{-T_{A}} \quad \cdots \\
& \cdot h_{n}\left(\tau_{1}, \tau_{2}, \ldots, \tau_{n}\right) \prod_{i=1}^{r} e\left(t, \tau_{i}\right) \prod_{i=1}^{n} d \tau_{i} \text {. }
\end{aligned}
$$

By substituting (40) in (2) we can write

$$
\begin{aligned}
u(t)=z_{0}(t)+ & \sum_{n=1}^{+\infty} \sum_{r=1}^{n} s^{n-r}(t)\left(\begin{array}{c}
n \\
r
\end{array}\right) \int \ldots \quad \ldots \int \\
& \cdot h_{n}\left(\tau_{1}, \tau_{2}, \ldots, \tau_{n}\right) \prod_{i=1}^{r} e\left(t, \tau_{i}\right) \prod_{i=1}^{n} d \tau_{i}
\end{aligned}
$$

where

$$
\begin{aligned}
z_{0}(t)=y_{0}+\sum_{n=1}^{+\infty} s^{n}(t) \int \cdots & \cdots \int h_{n} \\
& \cdots T_{A} \\
& \cdot\left(\tau_{1}, \tau_{2}, \ldots, \tau_{n}\right) \prod_{i=1}^{n} d \tau_{i}
\end{aligned}
$$

is the response of the system when the dynamic deviations are identically null; therefore, $z_{0}(t)$ represents the static characteristic of the device. Equation (42) shows that the quantity $z_{0}(t)$ can be expressed as a power series in the variable $s(t)$, where the coefficient of $s^{n}(t)$ coincides with the multiple integral of the corresponding $n$ th-order Volterra kernel.

Taking into account that there are no terms with index $r>n$ in the sum of (41), by inverting the order of summation and by substituting $r$ for $n$, we can deduce the following expression:

$$
\begin{array}{r}
u(t)=z_{0}(t)+\sum_{n=1}^{+\infty} \sum_{r=n}^{+\infty} s^{r-n}(t)\left(\begin{array}{l}
r \\
n
\end{array}\right) \int \ldots h_{r} h_{-T_{A}}^{+T_{B}} \ldots \int h_{r} \\
\cdot\left(\tau_{1}, \tau_{2}, \ldots, \tau_{r}\right) \prod_{i=1}^{n} e\left(t, \tau_{i}\right) \prod_{i=1}^{r} d \tau_{i}
\end{array}
$$

where the second term represents the output of a purely dynamic nonlinear system because it takes into account only the dynamic deviations of the signal within the interval $\left(t-T_{B}, t+T_{A}\right)$ around the actual instant $t$. Equation (43) can be rewritten synthetically as a modified Volterra series [12]

$$
u(t)=z_{0}(t)+\sum_{n=1}^{+\infty} z_{n}(t)
$$

where the quantity $z_{n}(t)$ represents the $n$ th-order term of the modified series and is given by

$$
\begin{aligned}
z_{n}(t)=\int \cdots g_{n}\left\{s(t) ; \tau_{1}, \ldots, \tau_{n}\right\} \\
\cdot \prod_{i=1}^{n}\left[\left(e\left(t, \tau_{i}\right)\right) d \tau_{i}\right] .
\end{aligned}
$$

This equation shows that the strictly dynamic phenomena are described by multidimensional convolution integrals of the signal dynamic deviations $e\left(t, \tau_{i}\right)=s\left(t-\tau_{i}\right)-s(t)$ with the $n$ th-order modified kernel $g_{n}\left\{s(t) ; \tau_{1}, \ldots, \tau_{n}\right\}$. By comparing (45) and (43), we can deduce that

$$
\begin{gathered}
g_{n}\left\{s(t) ; \tau_{1}, \ldots, \tau_{n}\right\}=h_{n}\left(\tau_{1}, \ldots, \tau_{n}\right)+\sum_{r=1}^{+\infty} s^{r}(t)\left(\begin{array}{c}
n+r \\
r
\end{array}\right) \\
\left.\cdot \int \begin{array}{c}
\ldots+T_{B} \\
\cdots \\
-T_{A}
\end{array}\right] h_{n+r}\left(\tau_{1}, \ldots, \tau_{n+r}\right) \prod_{i=1}^{r} d \tau_{n+i}
\end{gathered}
$$

where we have separated the term with $r=n$ and in the remaining terms we have substituted the generic index $r$ with $n+r$. Each modified kernel $g_{n}\left\{s(t) ; \tau_{1}, \ldots, \tau_{n}\right\}$ is nonstationary and symmetric with respect to its arguments; it is equal to the sum of the same order original Volterra kernel plus a sum of contributions directly related to higher-order kernels of the original Volterra series. Each $r$ th term of this summation is a function of both $s^{r}(t)$ and of $\tau_{1}, \ldots, \tau_{n}$, since it is obtained by integrating the original Volterra kernels of an order greater than $n$ in the space $d \tau_{n+1}, \ldots d \tau_{n+r}$ with $r \geq 1$. From (46), we can also see that, according to (45) and (3), $g_{n}\left\{0 ; \tau_{1} \cdots \tau_{n}\right\}=$ $h_{n}\left\{\tau_{1} \cdots \tau_{n}\right\}$ and consequently $z_{n}(t)=y_{n}(t)$ when $s(t)=0$. This means that the modified kernels coincide with the original Volterra ones when evaluated for $s(t)=0$. From (46), we can also see that $g_{r}\left\{0, \tau_{1}, \ldots, \tau_{r}\right\}=h_{r}\left(\tau_{1}, \ldots, \tau_{r}\right)$ and, consequently, according to (45) and (3), $z_{n}(t)=y_{n}(t)$, since $e(t, \tau)$ equal to $s(t-\tau)$ for $s(t)=0$.

When the system is linear with memory, all the kernels of the traditional Volterra series of order greater than one are null $\left(h_{r}\{\cdot\}=0\right.$ for $r \geq 2$ ). Therefore, from (42) we deduce that

$$
z_{0}(t)=y_{0}+s(t) \int_{-T_{A}}^{+T_{B}} h_{1}(\tau) d \tau .
$$

Furthermore, from (43), we obtain that also the kernels of the modified Volterra series of an order greater than one are null 
while the first order kernel is independent of $s(t)$ and coincides with the corresponding one of the traditional Volterra series, that is

$$
g_{1}\{s(t), \tau\}=h_{1}(\tau)
$$

while

$$
g_{r}\{\cdot\}=0 \quad \text { for } r \geq 2 .
$$

By substituting (47) and (48) in (8) and (6), we obtain the wellknown linear convolution integral

$$
\begin{aligned}
u(t)= & y_{0}+s(t) \int_{-T_{A}}^{+T_{B}} h_{1}(\tau) d \tau+\int_{-T_{A}}^{+T_{B}} \\
& \cdot h_{1}(\tau)[s(t-\tau)-s(t)] d \tau \\
= & y_{0}+\int_{-T_{A}}^{+T_{B}} h_{1}(\tau) s(t-\tau) d \tau .
\end{aligned}
$$

\section{REFERENCES}

[1] H. W. Chen, "Modeling and identification of parallel nonlinear systems: Structural classification and parameter estimation methods," Proc. IEEE, vol. 83, pp. 39-66, Jan. 1995.

[2] J. J. Bussgang, L. Ehrman, and J. W. Graham, "Analysis of nonlinear systems with multiple inputs,” Proc. IEEE, vol. 62, pp. 1088-1119, Aug. 1974.

[3] M. Schetzen, The Volterra and Wiener Theories of Non-Linear Systems. New York: Wiley-Interscience, 1980.

[4] J. S. Bendat, Non-Linear System Analysis \& Identification From Random Data. New York: Wiley, 1990.

[5] S. Boyd, Y. S. Tang, and L. O. Chua, "Measuring Volterra kernels," IEEE Trans. Circuits Syst., vol. 30, pp. 571-577, Aug. 1983.

[6] L. O. Chua and Y. Liao, "Measuring Volterra kernels (II)," Int. J. Circuit Theory Appl., vol. 17, no. <AU: ISSUE NO:?>, pp. 151-190, 1989.

[7] — "Measuring Volterra kernels (III): How to estimate the highest significant order," Int. J. Circuit Theory Appl., vol. 19, no. <AU: ISSUE NO:?>, pp. 189-209, 1991.

[8] C. Evans, D. Rees, L. Jones, and M. Weiss, "Probing signals for measuring nonlinear Volterra kernels," in Proc. Instrumentation and Measurement Technology Conf. (IMTC'95), Boston, MA, June 1995, pp. $10-15$.

[9] B. J. Leon and D. J. Schaefer, "Volterra series and Picard iteration of nonlinear circuits and systems," IEEE Trans. Circuits Syst., vol. CAS-25, pp. 789-792, Sept. 1978

[10] F. Filicori and G. Vannini, "Mathematical approach to large-signal modeling of electron devices," Electron. Lett., vol. 27, no. 4, pp. 357-359, 1991.

[11] F. Filicori, G. Vannini, and V. A. Monaco, "A nonlinear integral model of electron devices for HB circuit analysis," IEEE Trans. Microwave Theory Tech., vol. 40, pp. 1456-1465, July 1992.

[12] D. Mirri, G. Iuculano, F. Filicori, G. Vannini, G. Pasini, and G. Pellegrini, "A modified Volterra series approach for the characterization of nonlinear dynamic systems," in Proc. IEEE Instrumentation and Measurement Technology Conf. (IMTC'96), Brussels, Belgium, June 1996, pp. $710-715$.

[13] V. Volterra, The Theory of Functionals and of Integral and Integro-Differential Equations. New York: Dover, 1959.

[14] D. Mirri, G. Iuculano, F. Filicori, G. Pasini, and G. Vannini, "Modeling of non ideal dynamic characteristics in S/H-ADC devices," in Proc. IEEE Instrumentation and Measurement Technology Conf. (IMTC'95), Boston, MA, Apr. 1995, pp. 27-32.

[15] D. Mirri, G. Pasini, F. Filicori, G. Iuculano, G. Vannini, and G. Neri, "A nonlinear dynamic modeling approach for the characterization and error compensation in sampling digital instruments," in Proc. 14th IMEKO World Congress, vol. IVA, Tampere, Finland, June 1997, pp. 37-42.

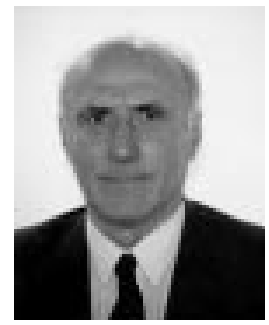

Domenico Mirri (M'91) was born in Imola, Italy in 1936. He received the M.S. degree in electronic engineering from the University of Bologna, Bologna, Italy, in 1963.

Currently, he is a Full Professor of electronic measurements at the University of Bologna. His current research interests are in the areas of digital measurement instruments, devices metrological characterization, and biomedical measurements.

[16] G. Vannini, "Non-linear integral modeling of dual-gate GaAs Mesfets," IEEE Trans. Microwave Theory Tech., vol. 42, pp. 1088-1091, June namic modeling of $\mathrm{S} / \mathrm{H}-\mathrm{ADC}$ devices," in Proc. IMEKO TC-4 Symp. ADC Modeling and Testing, Lisbon, Portugal, Sept. 2001, pp. 23-27.

[18] F. Filicori, G. Vannini, A. Santarelli, D. Torcolacci, and V. A. Monaco, "Accurate prediction of intermodulation distortion in GaAs MESFETS," 625-629.

19] G. Vannini, F. Filicori, and A. Santarelli, "Integral approaches to nonlinear modeling of electron devices," in Proc. Workshop Nonlinear Measurements and Modeling, 1997 IEEE MTT-S, Denver, CO, June 1997.

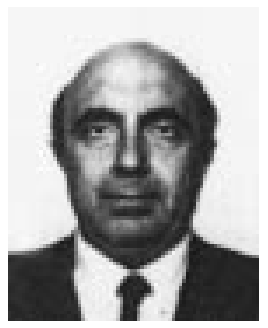

Gaetano Iuculano (M'66) received the degree in electronic engineering from the University of Bologna, Bologna, Italy.

He is currently a Professor of electrical measurements and metrology at the Department of Electronic Engineering of the University of Florence, Florence, Italy. He has experience in calibration applications and planning experiments, in reliability analysis and life testing for electronic devices and systems, and considerable expertise in practical statistical analysis for electrical engineering. He has authored and coauthored more than one hundred technical papers in his current research interests.

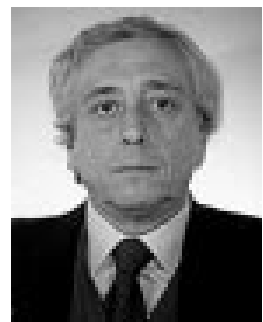

Fabio Filicori (M'98) was born in Imola, Italy in 1949. He received the degree in electronic engineering from the University of Bologna, Bologna, Italy, in 1964.

$\mathrm{He}$ is currently a Full Professor of applied electronics at the University of Bologna. His current research interests are in the areas of nonlinear circuit analysis and design, electronic devices modeling, digital measurement instruments, and

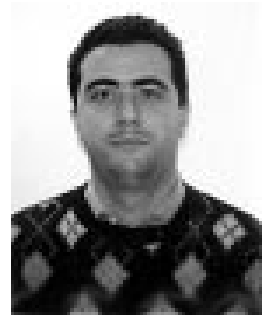

Gaetano Pasini (M'97) was born in Imola, Italy in 1964. He received the M.S. degree in electronic engineering from the University of Bologna, Bologna, Italy 1991.

Currently, he is with the University of Bologna, where he is Associate Professor in electrical measurement. His research activity is mainly oriented to digital signal processing in electronic instruments, power measurements, and characterization of nonlinear systems with memory. power electronics. 


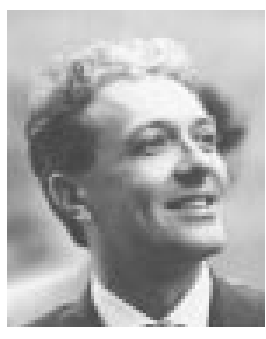

Giorgio Vannini (S'87-M'92) received the Laurea degree in electronic engineering and the Ph.D. degree in electronic and computer science engineering, from the University of Bologna, Bologna, Italy, in 1986 and 1992, respectively.

In 1992, he joined the Department of Electronics of the University of Bologna, as a Research Associate. Since November 1998, he has been an Associate Professor at the Faculty of Engineering, Dipartimento di Ingegneria, University of Ferrara, Ferrara, Italy. His research activity is mainly devoted to electron device modeling, CAD techniques for MMICs, and nonlinear circuit analysis and design.

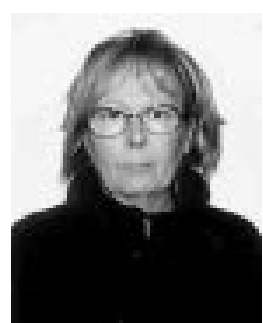

Gabriella Pellegrini Gualtieri graduated from the University of Rome, Rome, Italy, in 1965.

She is currently an Associate Professor of mathematical analysis at the University of Florence, Florence, Italy. Her research interests include numerical methods of nonlinear dynamic systems and uncertainty estimation in metrology by statistical techniques and probability theory. 